\title{
Low-loss polymer waveguides on nanoporous layers
}

\author{
G. Wicht, ${ }^{1}$ R. Ferrini, ${ }^{1,2, a)}$ S. Schüttel, ${ }^{3}$ and L. Zuppiroli ${ }^{1}$ \\ ${ }^{1}$ Laboratoire d'Optoélectronique des Matériaux Moléculaires, École Polytechnique Fédérale de Lausanne, \\ CH-1015 Lausanne, Switzerland \\ ${ }^{2}$ Centre Suisse d'Electronique et Microtechnique (CSEM), Entwicklungszentrum für Polytronics, \\ CH-4132 Muttenz, Switzerland \\ ${ }^{3}$ Functional Materials Group, ILFORD Imaging Switzerland GmbH, CH-1723 Marly, Switzerland
}

(Received 15 April 2011; accepted 17 September 2011; published online 10 October 2011)

\begin{abstract}
Multimode planar polymer waveguides were fabricated on hybrid polymer nanoporous layers with a fast industrial aqueous-based method well adapted to coat large-surface plastic substrates and easily scalable up to economical mass production volumes. Using a fiber probe internal light source technique, we were able to measure quantitatively the modal propagation losses. In particular, attenuation coefficients lower than $0.5 \mathrm{~dB} / \mathrm{cm}$ were found for the fundamental modes, thus showing the potential of our polymer nanoporous layers as optical isolation layers in waveguiding applications. (C) 2011 American Institute of Physics. [doi:10.1063/1.3647624]
\end{abstract}

Optical planar waveguides are fundamental building blocks in numerous optoelectronic devices, where signal transmission is needed. While inorganic materials have extensively been used to develop the best performing optical waveguides so far, in the last few years, the interest for organic materials has been rapidly growing. ${ }^{1}$ Polymer waveguides provide indeed a very attractive solution for the fabrication of optical systems characterized by low intrinsic losses, mechanical flexibility, and large surface, with the possibility of using simple, rapid, and cost-competitive production techniques. Moreover, the recent progresses in the material engineering of organic compounds make polymer multilayer films an ideal platform for the development of complex optical systems. ${ }^{2}$ In particular, several conventional polymers have been studied to fabricate optical waveguides: with poly(methyl methacrylate) (PMMA), for instance, waveguides were fabricated with propagation losses in the order of $0.1 \mathrm{~dB} / \mathrm{cm}$ or higher in the infrared spectral region. ${ }^{1}$ More recently, optimized optical polymers have been developed such as deuterated and halogenated polyacrylates, whose physical properties (refractive index, transparency, adhesion, surface energy, birefringence, etc.) and synthesis procedures are generally well mastered. With these latter polymers, monomode and multimode waveguides were fabricated with losses in the order of $0.01 \mathrm{~dB} / \mathrm{cm}$ in the infrared and red spectral intervals, respectively. Both fluorinated polyimides and perfluorocyclobutyl aryl ether polymers were also used to coat polymer-based waveguides with losses in the order of $0.1 \mathrm{~dB} / \mathrm{cm}$ in the infrared spectral region. Nevertheless, with the first materials, birefringence cannot be avoided and waveguide losses depend on polarization.

In general, the optical performances of planar waveguides strongly depend on the refractive index contrast $\Delta n$ between the core and the cladding layers, ${ }^{3}$ i.e., by increasing $\Delta n$, the light confinement in the guiding structure is improved, thus reducing the intrinsic contribution to propagation losses. In this respect, nanoporous layers have recently appeared as very attractive materials due to the pos-

\footnotetext{
${ }^{\text {a) }}$ Author to whom correspondence should be addressed. Electronic mail: rolando.ferrini@csem.ch.
}

sibility of achieving a very low refractive index $n$ (between 1.0 and 1.2) that can, thus, enhance $\Delta n$ in the existing waveguiding systems. More generally, these nanoporous layers are also being studied for applications in several other optical systems such as interferential anti-reflection coatigs ${ }^{4,5}$ and light out-coupling layers. ${ }^{6,7}$

As for optical planar waveguides, the possibility of introducing low- $n$ nanoporous layers in their architecture may eventually represent a very important break-through in the development of low-loss optical devices. However, while the beneficial role of nanoporous claddings has been extensively investigated in inorganic systems, ${ }^{8,9}$ their use in organic waveguide devices is still mostly unexplored. To this purpose, we remind that, if a refractive index contrast $\Delta n>0.2$ can be hardly achieved in all-polymer waveguides, the introduction of a low- $n$ nanoporous layer may give access to $\Delta n$ values larger than 0.3 , thus enabling a much better light confinement.

In this letter, we report on planar polymer waveguides, where a well-mastered and well known polymer, polyvinyl alcohol (PVA), is combined to hybrid polymer nanoporous layers with low refractive index that are used as optical isolation layers between the polymer core and the flexible plastic substrate. As illustrated in Ref. 10, such nanoporous claddings can be coated together with polymer layers over large surfaces by means of an industrial aqueous-based technology. With this latter technique, we fabricated multimode planar polymer waveguides on thick low-refractive index nanoporous layers, and we eventually characterized their optical performances by quantitatively measuring the modal propagation losses. The very low loss value $(\ll 1 \mathrm{~dB} / \mathrm{cm})$ obtained for the fundamental modes is lower than the values obtained with similar inorganic nanoporous systems ${ }^{8,9}$ and clearly demonstrates the potential of our nanoporous layers for optical isolation.

Planar polymer waveguides consisting of a thin (1.6 $\mu \mathrm{m})$ PVA core on top of a thick $(5 \mu \mathrm{m})$ nanoporous cladding layer were coated on a transparent $175 \mu$ m-thick poly(ethylene terephtalate) (PET) film (Fig. 1). In particular, the fabrication of the hybrid polymer nanoporous claddings starts from a coating solution consisting of a PVA suspension of $\mathrm{SiO}_{2}$ nanoparticles. ${ }^{10}$ Once deposited on the substrate, the solution is dried and a hybrid layer with a nanoporous 


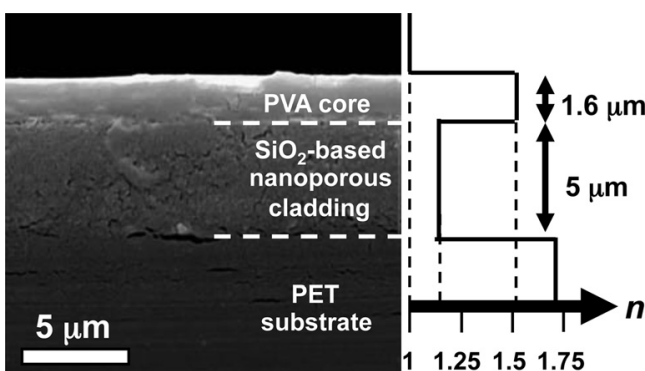

FIG. 1. SEM image of the cross section of a planar polymer waveguide coated on a PET substrate and consisting of a PVA core layer and a $\mathrm{SiO}_{2}$ based polymer nanoporous cladding. Both the thickness and the refractive index profile (at $575 \mathrm{~nm}$ ) of the waveguide components are reported.

morphology is formed: the primary nanoparticles form larger agglomerates in the order of tens to hundreds nanometer with the PVA acting as a simple binder, while air pores appear in the layer bulk. The resulting porosity and the pore size are the critical factors that set the optical properties of such films. On one hand, the composition of the coating solutions was chosen to minimize the PVA content (i.e., to maximize the film porosity and minimize $n$ ) while keeping an acceptable mechanical stability. On the other hand, since the length scales of the porous structure are much lower than the visible wavelengths (i.e., less than hundreds nanometer) and the $\Delta n$ between the $\mathrm{SiO}_{2}$ and the film matrix is very low, the light scattering from the nanoparticle agglomerates dispersed in the layer bulk is very moderate. Therefore, by properly controlling the nanoparticle surface, the solution composition, the fabrication process, and eventually, the film thickness, highly transparent (transmission higher than 90\%) nanoporous films were obtained with a very low average refractive index $(n<1.2)$ in the visible spectral region. ${ }^{10}$ Such $\mathrm{SiO}_{2}$-based layers were combined with simple PVA films and coated on the PET substrate with a precise control over the layer thickness and the optical properties. Details on the both the composition of the coating solutions as well as on the coating technique are given in Ref. 10.

In order to characterize the bulk and the interface morphology of both the PVA core and the polymer nanoporous cladding layer, scanning electron microscopy (SEM) micrographs were taken. ${ }^{10}$ The SEM image of a typical planar polymer waveguide is shown in Fig. 1. On one hand, the SEM analysis reveals the homogeneously porous morphology of the cladding layer with pore sizes in the order of several tens nanometer, i.e., well below the 100-nm threshold where the visible light starts to be scattered. On the other hand, a slight roughness (approximately tens to hundreds nanometer) appears at both the core- and substrate-cladding interfaces due to the presence of nanoparticle agglomerates at the surface of the nanoporous film. These latter agglomerates can actually originate a moderate light scattering in the order of few percentages. ${ }^{10}$ However, due to the high dilution of the coating solution, when the PVA film is coated on top of the slightly rough dried cladding, the induced roughness is partly leveled out without replication and the core top surface results to be almost flat.

Ellipsometry was used to measure the thickness and the optical properties of both the polymer and the nanoporous layers. ${ }^{10}$ The values 5 and $1.6 \mu \mathrm{m}$ were obtained for the clad- ding and the core thicknesses, respectively (Fig. 1). The average $n$ values at $575 \mathrm{~nm}$ for the PET substrate, the $\mathrm{SiO}_{2}$ based nanoporous cladding layer, and the PVA core $(n=1.7$, 1.17, and 1.52, respectively) are reported in Fig. 1.

In order to characterize the optical performances of our polymer waveguides, we adopted a fiber probe internal light source technique, ${ }^{11-13}$ which measures the intensity attenuation of the light diffracted along the waveguide. As internal light source, ${ }^{14}$ we used the light emission from organic fluorescent dyes locally adsorbed onto the polymer core. In particular, ink-jet printing was used to deposit a few drops of a water solution of 4-[(4-dimethylamino)styryl]-1-methylpyridium p-toluenesulfonate [DSM: see Fig. 2] on a selected area $\left(\sim 4 \mathrm{~mm}^{2}\right)$ on the PVA layer. We highlight the fact that, since the dye molecules are localized in a selected area (i.e., the light injection region), while the rest of the waveguide, where the guided signal propagates, remains undoped, the system is free from reabsorption losses. Therefore, the core and the cladding layers being highly transparent, ${ }^{10}$ the main extrinsic contribution to propagation losses comes from the interface roughness (Fig. 1). ${ }^{3}$ A typical set of scattered fluorescence spectra measured at different distances from the injection point (i.e., the DSM-doped region) is shown in Fig. 3.

In Fig. 4 , the intensity of light emitted at $\lambda_{\mathrm{em}}=575 \mathrm{~nm}$ and scattered out of the waveguide plane is plotted as a function of the distance $\mathrm{L}$ with a spatial interval $\Delta \mathrm{L}=2 \mathrm{~mm}$. In particular, the quantity $\log (\mathrm{T})=\log _{10}\left(\mathrm{I}_{\mathrm{L}} / \mathrm{I}_{0}\right)$ is reported (gray squares), where $\mathrm{I}_{\mathrm{L}}$ and $\mathrm{I}_{0}$ are the intensities detected at the distance $\mathrm{L}$ and at the injection point, respectively. On one hand, since, in presence of losses, the light propagation in an optical waveguide follows the Lambert-Beer $\operatorname{law}, \log (\mathrm{T})$ is expected to decay linearly with $\mathrm{L}$ with a gradient equal to the attenuation coefficient $\alpha=-(10 / \mathrm{L}) \times \log (\mathrm{T}) \mathrm{dB} / \mathrm{cm}{ }^{3}$ On the other hand, due to the multimode nature of our waveguides, several linear regimes can be identified in Fig. 4. At a given distance L, several modes are propagating through the waveguide and $\alpha=\Sigma \alpha_{i}$, where $\alpha_{i}$ 's are the modal attenuation coefficients. Moving away from the injection point, any time a high-order mode is completely lost, the gradient of the decay curve decreases since only low-order (i.e., less lossy) modes keep propagating. In order to estimate the modal losses, we fitted the experimental data of Fig. 4 with a standard mode solving model introducing a phenomenological loss parameter

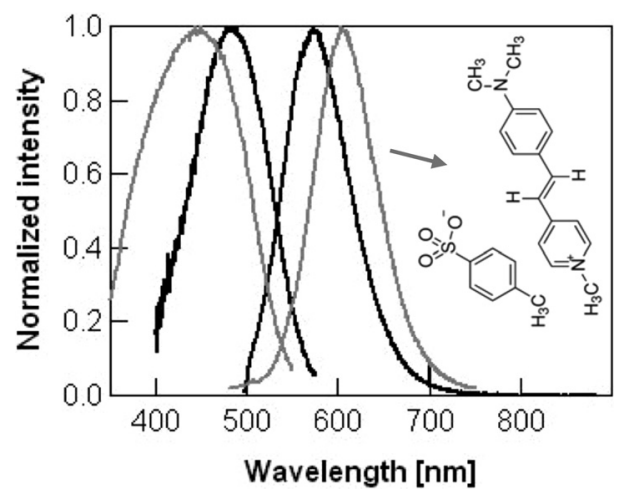

FIG. 2. Gray lines: Excitation (emission wavelength $=611 \mathrm{~nm}$ ) and fluorescence (excitation wavelength $=454 \mathrm{~nm}$ ) spectra of a water solution of DSM (see the inset). Black lines: Corresponding absorption and emission (excitation wavelength $=457 \mathrm{~nm}$ ) spectra of a PVA layer doped with DSM molecules. 


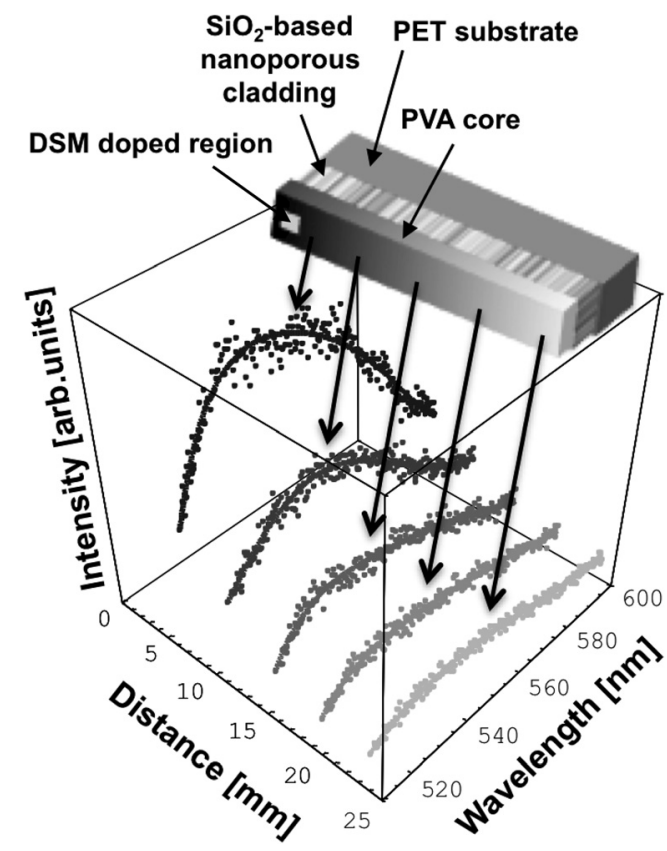

FIG. 3. Typical set of scattered fluorescence spectra measured at different distances L from the injection point (i.e., the DSM-doped region). The best fit curves (solid lines) of the experimental data (dots) are shown as reference.

$k$ in the nanoporous cladding to mimic the extrinsic loss contribution related to the interface roughness. By measuring the light scattering at large distances $(\mathrm{L}>50 \mathrm{~mm})$, we verified that the gradient of the linear section I in Fig. 4 (gray solid line) remains constant, thus showing that at $\mathrm{L}=35 \mathrm{~mm}$, all high-order modes are lost and only the fundamental transverse electric (TE0) and magnetic (TM0) modes are still propagating with an overall attenuation coefficient $\alpha_{\mathrm{I}}=0.7 \mathrm{~dB} / \mathrm{cm}$. Moreover, the best fit of these latter data yielded $k=1.23 \times 10^{-4}$ and the modal attenuation coefficients $\alpha_{\mathrm{TE} 0}=0.26 \mathrm{~dB} / \mathrm{cm}$ and $\alpha_{\mathrm{TM} 0}=0.44 \mathrm{~dB} / \mathrm{cm}$. Finally, all data reported in Fig. 4 could be fitted with a very good agreement between the experimental and the theoretical attenuation factors. In particular, three other propagation sections could be identified (gray solid lines): section II $(20<\mathrm{L}<35 \mathrm{~mm}$ and $\alpha_{\text {II }}=1.8 \mathrm{~dB} / \mathrm{cm}$ ), where the first order TE1 mode is propagating along with the fundamental TE0 and TM0 modes

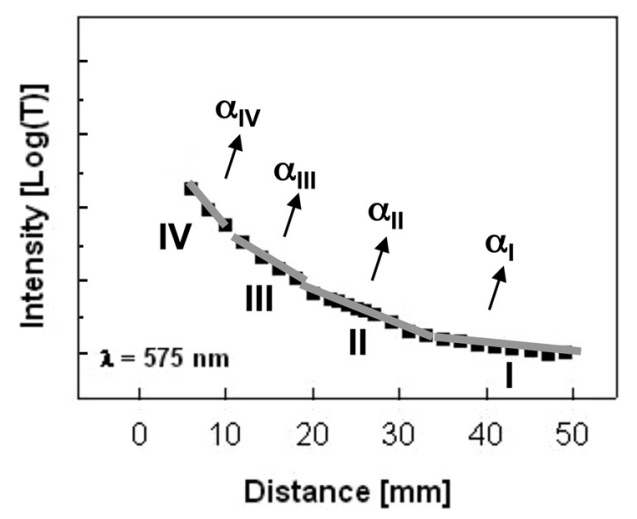

FIG. 4. Intensity $\left[\log (T)=\log _{10}\left(\mathrm{I}_{\mathrm{L}} / \mathrm{I}_{0}\right)\right]$ of light emitted at $\lambda_{\mathrm{em}}=575 \mathrm{~nm}$ and scattered out of the waveguide plane as a function of the distance $\mathrm{L}$ (black squares). The data are derived from the spectra shown in Fig. 4. The best fit of the experimental data (gray lines) as well as the overall attenuation coefficients $\alpha_{\mathrm{I}, \mathrm{II}, \mathrm{III}, \mathrm{IV}}$ in the corresponding sections are reported.
$\left(\alpha_{\mathrm{TE} 1}=1.1 \mathrm{~dB} / \mathrm{cm}\right)$, section III $\left(5<\mathrm{L}<20 \mathrm{~mm}\right.$ and $\alpha_{\text {III }}=3.7$ $\mathrm{dB} / \mathrm{cm}$ ), where the first order TM1 mode is still propagating $\left(\alpha_{\mathrm{TM} 1}=1.9 \mathrm{~dB} / \mathrm{cm}\right)$, and section IV $\left(\mathrm{L}<5 \mathrm{~mm}\right.$ and $\alpha_{\mathrm{IV}}=6.6$ $\mathrm{dB} / \mathrm{cm}$ ), where the second order TE2 mode adds up to the previous modes $\left(\alpha_{\mathrm{TE} 2}=2.9 \mathrm{~dB} / \mathrm{cm}\right)$.

In conclusion, we fabricated planar polymer waveguides by coating a PVA core film on a $\mathrm{SiO}_{2}$-based polymer nanoporous layer with a fast large-area industrial aqueous-based method easily scalable up to economical mass production volumes. Using a fiber probe internal light source technique, we were able to measure the modal propagation losses. In particular, attenuation coefficients well below $0.5 \mathrm{~dB} / \mathrm{cm}$ were found for the fundamental modes. This latter value well matches the best results obtained with inorganic systems so far, ${ }^{8,9}$ thus demonstrating the potential of our hybrid nanoporous layers as optical isolation films both for waveguides, where very low losses are usually demanded, and for technologies with less critical loss requirements (e.g., multicolor displays). Finally, we highlight that, on one hand, our coating method can be easily scaled up to single-step fabrication techniques, such as the curtain coating, that allow both the coating of very large surfaces (from $\mathrm{cm}^{2}$ to several $\mathrm{m}^{2}$ ) and a better control of the interface quality, i.e., a further reduction of the propagation losses. On the other hand, thin core layers may be coated with this latter industrial technique to fabricate monomode waveguides.

The authors thank M. Schär and M. Longchamp from the École Polytechnique Fédérale de Lausanne (EPFL, Switzerland) and G. Liechti and J. Ketterer from Ilford Imaging Switzerland GmbH (Marly, Switzerland) for their help. This work was supported by the Swiss Confederation Innovation Promotion Agency (Commission pour la Technologie et l'Innovation-CTI) and the Swiss National Science Foundation (SNSF) through the CTI-8184.1 EPRP-IW and the SNSF-200021-111856 projects, respectively.

${ }^{1}$ H. Ma, A. K.-Y. Jen, and L. R. Dalton, Adv. Mater. 14, 1339 (2002).

${ }^{2}$ T. Hanemann, B. Schumacher, and J. Hausselt, Microelectron. Eng. 87, $533(2010)$

${ }^{3}$ B. E. A. Saleh and M. C. Teich, Fundamentals of Photonics (Wiley, New York, 1991).

${ }^{4}$ J.-Q. Xi, M. F. Schubert, J. K. Kim, E. F. Schubert, M. Chen, S.-Y. Lin, W. Liu, and J. A. Smart, Nat. Photonics 1, 176 (2007).

${ }^{5}$ M. Yamaguchi, H. Nakayama, K. Yamada, and H. Imai, Opt. Lett. 34, 2060 (2009).

${ }^{6}$ T. Tsuitsui, M. Yahiro, H. Yokogawa, K. Kawano, and M. Yokoyama, Adv. Mater 13, 1149 (2001).

${ }^{7}$ H. J. Peng, Y. L. Ho, X. J. Yu, and H. S. Kwok, J. Appl. Phys. 96, 1649 (2004).

${ }^{8}$ H. F. Arrand, T. M. Benson, P. Sewell, A. Loni, R. J. Bozeat, R. Arens-Fischer, M. Krüger, M. Thönissen, and H. Lüth, IEEE J. Sel. Top. Quantum. Electron. 4, 975 (1998).

${ }^{9}$ S. S. Ponoth, N. T. Agarwal, P. D. Persans, and J. L. Plawsky, J. Vac. Sci. Technol. B 22, 902 (2004).

${ }^{10}$ G. Wicht, R. Ferrini, S. Schüttel, and L. Zuppiroli, Macromol. Mater. Eng. 295, 628 (2010)

${ }^{11}$ N. Nourshargh, E. M. Starr, N. I. Fox, and S. G. Jones, Electron. Lett. 21, 818 (1985).

${ }^{12}$ M. D. Himel and U. J. Gibson, Appl. Opt. 25, 4413 (1986).

${ }^{13}$ B. Lombardet, R. Ferrini, L. A. Dunbar, R. Houdré, C. Cuisin, O. Drisse, F. Lelarge, F. Pommereau, F. Poingt, and G.-H. Duan, Appl. Phys. Lett. 86, 111111 (2005).

${ }^{14}$ R. Ferrini, D. Leuenberger, M. Mulot, Q. Min, R. Moosburger, M. Kamp, A. Forchel, S. Anand, and R. Houdre, J. Quantum Electron. 38, 786 (2002). 